\title{
Les femmes ne s'intéressent pas seulement à la médecine de famille
}

\author{
Nora Bienz \\ Dr méd., Médecine interne générale FMH, présidente de l'ASMAC Berne, membre du Comité directeur de I'ASMAC Suisse, Spiez
}

Certaines déclarations ne nécessitent pas de grande réflexion pour constater leur contenu amer. «Contrairement à leurs collègues masculins, les femmes s'intéressent avant tout à la pédiatrie et à la médecine de famille [...] Dans les disciplines chirurgicales comme l'orthopédie, il n'y a que 10\% de femmes, cela les intéresse moins» [1]. Or ce sont de tout autres raisons que le manque d'intérêt des femmes qui conduisent à cette situation insatisfaisante.

Les femmes médecins exercent plus rarement dans des disciplines impliquant une activité chirurgicale. Cette partie de la déclaration du président de la FMH, Jürg Schlup, dans l'émission télévisée «10vor10» du 29 octobre 2018 est juste. Par contre, son explication selon laquelle les femmes s'intéresseraient moins aux tâches chirurgicales est fausse. Les raisons du choix d'une discipline sont multiples. Un regard sur les débuts de mon propre parcours professionnel en offre un exemple parlant.

\section{De l'euphorie à la désillusion}

J'ai passé mon examen fédéral à l'Université de Berne en 2011. Je m’intéressais surtout à la gynécologie opératoire, discipline sur laquelle j'avais déjà orienté mon année d'étude à option. J'ai choisi mon premier poste dans le service de chirurgie viscérale d'un hôpital de taille moyenne. J'aimais particulièrement mon travail et j'ai développé une véritable passion pour la chirurgie. Ma fascination restait intacte, même s'il fallait se tenir au milieu de la nuit à la table d'opération. Pour moi, travailler 24 heures d'affilée était un défi au sens positif du terme. Je considérais le service du week-end entre deux semaines de travail normal comme un mal nécessaire pour pouvoir opérer. Mais en l'espace d'une année, mon euphorie des débuts s'est passablement réduite. J'aspirais à plus de temps et d'énergie pour ma vie privée et mes intérêts extraprofessionnels.

\section{Le travail en équipe au lieu de semaines de 12 jours}

Lors de mon entretien d'embauche pour mon engagement consécutif en gynécologie, on m’a notamment demandée si jétais disposée à mettre ma vie privée «en veilleuse». C'est à ce moment-là que j'ai commencé à réfléchir à mon avenir et à me demander si une carrière chirurgicale était vraiment un objectif souhaitable. A l'époque, je n'avais pas de bons exemples à suivre parmi les chirurgiens-cadres. Je ne voyais pas non plus de perspectives d'amélioration des conditions de travail et je savais que je voudrais, tôt ou tard, fonder une famille.

Comme je n'ai pas pu obtenir immédiatement le poste en gynécologie, j'ai eu la possibilité de travailler pendant six mois aux soins intensifs. Après l'année en chirurgie avec des services de 12 jours, d'innombrables services de piquet et plus de 400 heures supplémentaires, j'ai vécu comme reposant le système du travail en équipe très bien organisé des soins intensifs, avec une semaine de travail de 50 heures conforme à la loi. Après mûre réflexion et d'innombrables discussions, j'ai finalement abandonné l'idée de faire de la gynécologie opératoire. J'ai prolongé mon contrat aux soins intensifs et cherché un emploi consécutif dans un service de médecine interne de catégorie A. J'y ai trouvé une journée de travail relativement bien organisée, une hiérarchie horizontale, une formation postgraduée bien structurée, de l'encouragement et un bon travail d'équipe. En 2017, j'ai finalement obtenu le titre de spécialiste en médecine interne générale et acquis mes premières expériences en tant que cheffe de clinique. Actuellement, je suis ma deuxième spécialisation en médecine intensive. Jusqu'ici, je n'ai pas regretté mon choix.

\section{Les femmes dans l'impasse}

Beaucoup de mes collègues étudiantes sont aujourd'hui médecins spécialistes ou sur le point de terminer leur formation postgraduée. Quelques-unes parmi elles ont 


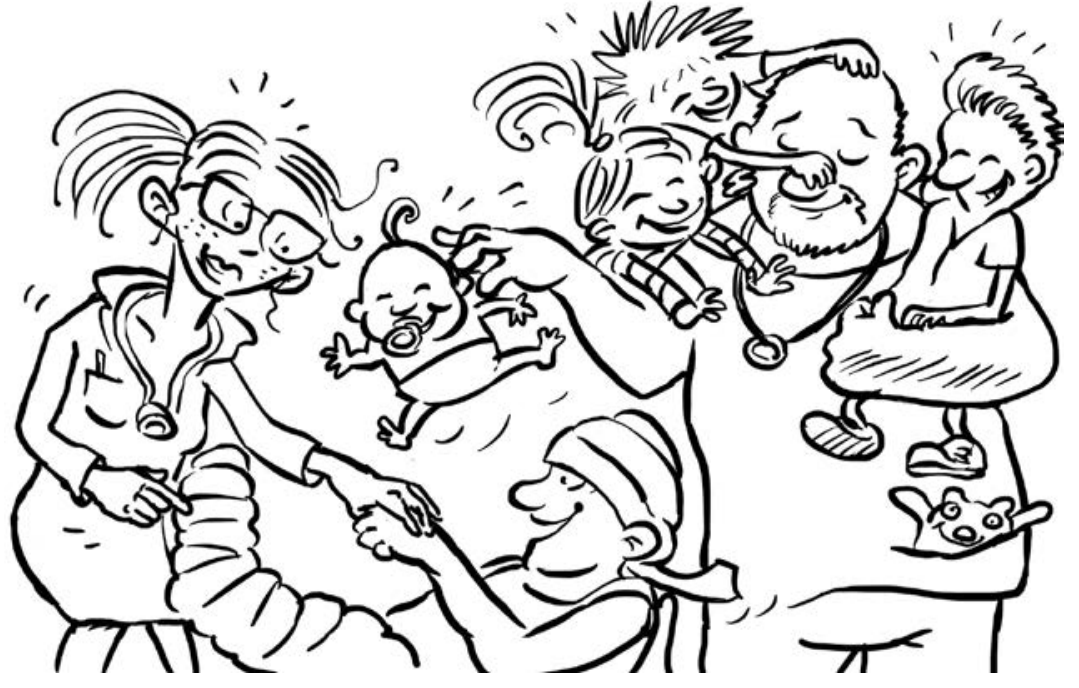

poursuivi leur parcours chirurgical. Hélas, je constate dans mon entourage l'augmentation du nombre de femmes médecins qui, malgré leur engagement, leur talent, leur passion pour leur profession et une formation postgraduée (presque) terminée, n'avancent pas. Les jeunes femmes médecins spécialistes ne sont pas suffisamment encouragées dans les hôpitaux ou n'obtiennent pas de promotion et choisissent, dans l'urgence, une autre voie. Les femmes trentenaires, c'est-à-dire en âge de fonder une famille, rencontrent en particulier beaucoup de difficultés dans l'atteinte de leurs objectifs de carrière. Cela se traduit aussi par les chiffres: d'après une récente analyse de données réalisée sur mandat du Conseil-exécutif du canton de Berne [2], à l'Hôpital de l'Ile, le plus grand employeur du secteur hospitalier du canton, $14 \%$ seulement des postes de cadre sont occupés par des femmes.

Pourquoi les femmes préfèrent-elles s'installer comme pédiatre ou généraliste? Et quelles solutions envisage-t-on pour pourvoir davantage de postes de cadre dans les cliniques avec des femmes?

\section{Plus de travail à temps partiel - plus de temps pour la famille}

Celui qui souhaite connaître les causes de cette évolution n'a pas besoin de chercher longtemps. De toute évidence, deux facteurs essentiels déterminent le choix de la discipline et les ambitions de carrière: la possibilité de travailler à temps partiel et l'offre de places de travail favorables à la famille. Il est évident que la compatibilité entre profession et vie de famille est un sujet fondamental quand il s'agit des perspectives professionnelles des femmes médecins.

Un nouveau projet de l'association faîtière ASMAC s'inscrit dans cette idée. Il a pour objectif d'encourager le travail à temps partiel dans les hôpitaux suisses. Un "plan de construction, temps partiel» a été élaboré sur la base des résultats d'un sondage et des éclaircissements obtenus dans différents hôpitaux suisses. Cela doit permettre de réaliser dès cette année des solutions sur mesure pour les cliniques et services. Elles permettront ensuite d'adapter l'organisation d'une clinique ou d'un service de façon à pouvoir augmenter le nombre de postes à temps partiel.

\section{Durée de travail: 50 heures et plus...}

D'autres motifs expliquent la faible part des femmes dans les disciplines chirurgicales et les positions de cadre. Ils sont en partie similaires à ceux que l'on trouve dans d'autres branches: la répartition traditionnelle des rôles dans les familles suisses, le manque d'enthousiasme des employeurs à s'accommoder de congés maternité, mais aussi les structures et réseaux d'entreprises dominés par les hommes. Néanmoins, il existe aussi des facteurs spécifiques aux médecins. Il s'agit notamment:

- des structures hospitalières avec leurs conditions de travail,

- de l'énorme part du temps consacré à des activités non médicales,

- de la durée de travail normale élevée combinée au non-respect de la loi sur le travail.

La durée hebdomadaire de travail des médecins-assistants est une particularité de la formation médicale postgraduée en Suisse. Avec ses 50 heures, elle est plus élevée que dans les pays voisins. Il est donc logique qu'avec une durée de travail de cette ampleur, même un engagement à $80 \%$ (c'est-à-dire 40 heures par maine) représente encore une charge considérable pour une famille. De plus, un médecin-assistant sur deux travaille en réalité plus que les 50 heures par semaine admises par la loi, avec toutes les conséquences négatives que cela implique pour son propre bien-être, le bien-être de la famille et la sécurité des patients.

\section{Moins de bureaucratie, plus de temps pour les malades}

A première vue, cela ne semble pas être une idée très originale. Pourtant, c'est à cause de cette énorme charge de travail qu'il vaut la peine de faire avancer les mesures visant à simplifier les déroulements administratifs dans les hôpitaux. Car d'après une étude actuelle de l'Université de Lausanne [3], les médecins hospitaliers ne peuvent plus consacrer qu'un tiers de leur temps de travail aux malades. L'ASMAC tente de remédier à cette situation avec sa campagne intitulée «Plus de médecine et moins 
de bureaucratie!» (www.plus-de-médecine-moins-debureaucratie.ch). Les exemples mis en œuvre avec succès montrent qu'il est judicieux de déléguer certaines tâches administratives à certains professionnels spécialement formés à cet effet, ou du moins de les simplifier.

La réduction des tâches administratives accomplies par les médecins bénéficie d'une part aux patients et contribue d'autre part à utiliser plus efficacement le temps disponible à l'hôpital. Moins d'administration peut aussi se traduire par davantage de temps pour les opérations et la formation. Une activité chirurgicale demande beaucoup d'entraînement. L'étude lausannoise [3] mentionnée révèle que $6 \%$ seulement du temps de travail est disponible pour la formation. Passer plus de temps à l'hôpital et en consacrer si peu à la formation n'est pas la recette du succès pour devenir une excellente femme médecin. Une réduction de la bureaucratie est une mesure bien plus efficace pour parvenir à ce but que la formation de nuit de médecins épuisés par les heures supplémentaires.

\section{Encourager et exiger}

Le travail scientifique ou son encouragement est une autre condition pour atteindre un poste de cadre en médecine. En raison de la forte charge de travail en clinique, il n'est pas rare qu'il soit exigé pendant les loisirs, ce qui est incompatible avec la vie de famille, indépendamment du sexe. Cette approche semble aussi douteuse pour ce qui concerne la qualité de la recherche. L'extrême complexité de la démarche pour obtenir des fonds destinés à la recherche suppose une réduction des tâches cliniques - du moins pour les femmes médecins avec des ambitions de carrière. De plus, nous devons nous demander s'il n'est pas possible de davantage séparer la recherche et la clinique. Nous avons autant besoin de femmes médecins compétentes dans le domaine clinique que de bonnes chercheuses. Mais est-il vraiment nécessaire d'être bon dans les deux domaines pour occuper un poste de cadre?

\section{Le problème des médecins-chefs}

A ce titre, il faut finalement aussi mentionner une particularité du secteur hospitalier. Je parle des structures hiérarchiques qui conduisent à la forte dépendance des médecins en formation. Les décisions concernant des congés pour la recherche ou l'octroi d'une bourse dans une clinique partenaire à l'étranger sont souvent prises par une seule personne au niveau de la direction.

Si le/la supérieur/e hiérarchique rejette la demande d'une personne, cette dernière aura des difficultés à avancer. Et si le chef considère que les modèles de tra- vail à temps partiel ne sont pas réalisables, il devient difficile d'accomplir un parcours clinico-scientifique tout en assumant des obligations familiales. Notamment dans les disciplines chirurgicales, l'encouragement direct par les supérieurs hiérarchiques se répercute sur la vitesse à laquelle le catalogue des opérations se remplit. A cela s'ajoute que le paysage hospitalier en Suisse est relativement petit dans le domaine de la médecine de pointe. Il n'est pas rare que le refus d'un chef entrave ensuite l'avancement dans un autre hôpital. Pour établir une culture de l'encouragement et de la promotion équitable, il faut donc des instances décisionnelles bénéficiant d'un large appui. Pour prendre des décisions, ces dernières doivent s'appuyer sur des critères objectifs tels que les attestations de formation complémentaire, les connaissances linguistiques, l'expérience professionnelle, les publications scientifiques, mais aussi d'autres facteurs comme la compétence sociale et la capacité à travailler en équipe.

\section{Un changement de culture est nécessaire}

A l'heure actuelle, environ $60 \%$ des médecins fraîchement diplômés sont des femmes et la tendance est à la hausse. Il est donc vraiment temps de réfléchir aux perspectives à long terme de ces femmes dans la profession, car les femmes médecins qui quittent prématurément la vie active accentuent la pénurie de spécialistes. Nous ne souhaitons pas seulement maintenir dans la profession la relève féminine bien formée à grands frais, nous voulons que les femmes médecins puissent faire carrière. Le choix de la discipline ou la fondation d'une famille ne doivent pas être un obstacle dans cette démarche.

Des conditions de travail flexibles et favorables à la famille pour les femmes et les hommes, une adaptation des structures internes dans les cliniques/services et un changement de culture sont des conditions indispensables pour que les femmes médecins puissent choisir leur discipline sans préjugés et en fonction de leurs propres intérêts. Tel est notre objectif.

\section{Crédit photo}

Nicolas d'Aujourd'hui

\section{Références}

1 Emission 10vor10, 29 octobre 2018, https://www.srf.ch/play/ tv/10vor10/video/10vor10-vom-29-10-2018?id=2f0198e2-ac24 4e9f-9ca0-5fof9d52e385.

2 Interpellation, réponse du Conseil-exécutif du canton de Berne, Discrimination à l'Hôpital de l'Ile: il faut améliorer l'égalité dans les cliniques et hôpitaux privés et publics du canton de Berne! $\mathrm{N}^{\circ}$ de l'affaire 2018.RRGR.190.

3 Wenger N, Méan M, Castioni J, Marques-Vidal P, Waeber G, Garnier A. Allocation of Internal Medicine Resident Time in a Swiss Hospital: A Time and Motion Study of Day and Evening Shifts. Annals.org; 2017. 